\title{
Density Dependency of Tempered Martensite Hardness in Sintered Carbon Steel
}

\author{
Singon Kang ${ }^{1}$, Minwook Kim ${ }^{2}$ and Seok-Jae Lee ${ }^{2, *}$ \\ ${ }^{1}$ Advanced Steel Processing and Products Research Center, Colorado School of Mines, \\ Golden, CO 80401, USA \\ ${ }^{2}$ Division of Advanced Materials Engineering, Research Center for Advanced Materials Development, \\ Chonbuk National University, Jeonju 561-756, Republic of Korea
}

The density dependence of tempered martensite hardness was studied and we have proposed a new equation to predict the hardness of tempered martensite sintered carbon steel. This was achieved by considering the density-dependent tempering value in the tempering parameter. The existence of porosity influenced both the decrease in tempered martensite hardness and the decrease in the activation energy for tempering, resulting in a lower tempering parameter. The variation of the hardness of tempered martensite predicted by the proposed equation was in good agreement with experimental data obtained under various tempering conditions and relative densities. [doi:10.2320/matertrans.M2015090]

(Received April 3, 2015; Accepted May 11, 2015; Published June 19, 2015)

Keywords: tempered martensite hardness, tempering parameter, density, activation energy, carbon steel

\section{Introduction}

A tempering process is performed in quenched martensitic steels to improve their toughness and ductility. However, these processes also lead to a reduction in martensite hardness. The variation of mechanical properties of tempered martensitic steels is primarily dependent on the tempering conditions such as tempering temperature and holding time. The effect of tempering process conditions on the evolution of the hardness values in martensitic steels is usually evaluated by a tempering parameter. ${ }^{1)}$ The tempering parameter can also be used for evaluating other mechanical properties such as yield and tensile strength and toughness after tempering. ${ }^{2,3)}$ The tempering process is also affected by the chemical composition of the martensitic steels; tempering is strongly related to the diffusion of carbon atoms in the martensite., ${ }^{4,5)}$ For this reason, previous studies in the literature have used different $C$ value, which is a material constant incorporated in the tempering parameter, to modify the tempering parameter for different chemical compositions. ${ }^{6,7)}$ The present authors have proposed an empirical equation to obtain the composition-dependent tempering parameter in low alloy steels with reliable accuracy between calculated and measured hardness values. ${ }^{8)}$

The tempering process is also applicable in sintered and quenched carbon and alloy steels with dispersed pores, as fabricated by powder metallurgy (PM). PM is one of the most attractive candidates for the processing of metals. ${ }^{9)}$ In particular, sintered automotive parts made from carbon and alloy steels using PM processes are very attractive due to several advantages: ${ }^{10-12)}$ (1) lower density due to a higher porosity, which contributes to a reduction in weight; (2) higher precision in shape and in dimension of steel components, which decreases the need for extra processing steps causes by unexpected distortions after heat treatments; and (3) precise control of the alloying composition. Conventional PM processes for metallic materials include powder

*Corresponding author, E-mail: seokjaelee@jbnu.ac.kr mixing, compacting, and sintering. Each of these results in the formation of voids in the PM processed specimens. The void characteristics including void size, shape, and distribution, mainly affect the mechanical properties of the PM processed specimens.

Although tempering is an essential process for sintered and quenched automotive steel parts, detailed information about the tempering behavior of PM-processed steels is lacking. A key aspect for the tempering of PM steels is the relationship between the density which is directly influenced by porosity and tempering behavior. In the present study, we investigated the martensite hardness of PM-processed carbon steel under diverse tempering conditions. The effect of density on the tempered martensite hardness was systematically analyzed by comparing the hardness values between sintered specimens with pores and fully dense specimens. In particular, the density effects on both the activation energy of tempering and the tempering parameter are discussed in detail.

\section{Experimental Procedure}

Commercially pure elemental powders, including Fe, Mn, $\mathrm{Si}$, and graphite, were prepared to fabricate an alloy with a chemical composition of $\mathrm{Fe}-0.55 \mathrm{C}-0.67 \mathrm{Mn}-0.21 \mathrm{Si}$ (in mass\%), which corresponds to JIS S55C steel. ${ }^{13)}$ The designed mass of the $\mathrm{Fe}, \mathrm{Ni}, \mathrm{Cr}, \mathrm{Mo}$, and graphite powders were precisely weighed and mixed in a tubular mixer for $3 \mathrm{~h}$ to obtain a homogenized powder mixture. The homogeneously mixed alloy powder was compacted with a pressure of $850 \mathrm{MPa}$ using a hydraulic press to produce cylindrical green compacts with a diameter of $11 \mathrm{~mm}$ and a length of $10 \mathrm{~mm}$. The green compacts were sintered at $1150^{\circ} \mathrm{C}$ for 30 min under a $\mathrm{N}_{2}+10 \% \mathrm{H}_{2}$ atmosphere, followed by furnace cooling to room temperature. The average density of the sintered specimens, as measured by the Archimedes method, was as $6.9 \mathrm{~g} / \mathrm{cm}^{3}$. This is approximately $90 \%$ of the bulk specimen density. The theoretical density of ferritic Febased alloys at room temperature $\left(\mathrm{g} / \mathrm{cm}^{3}\right)$ can be calculated using the following equation: ${ }^{14}$ ) 

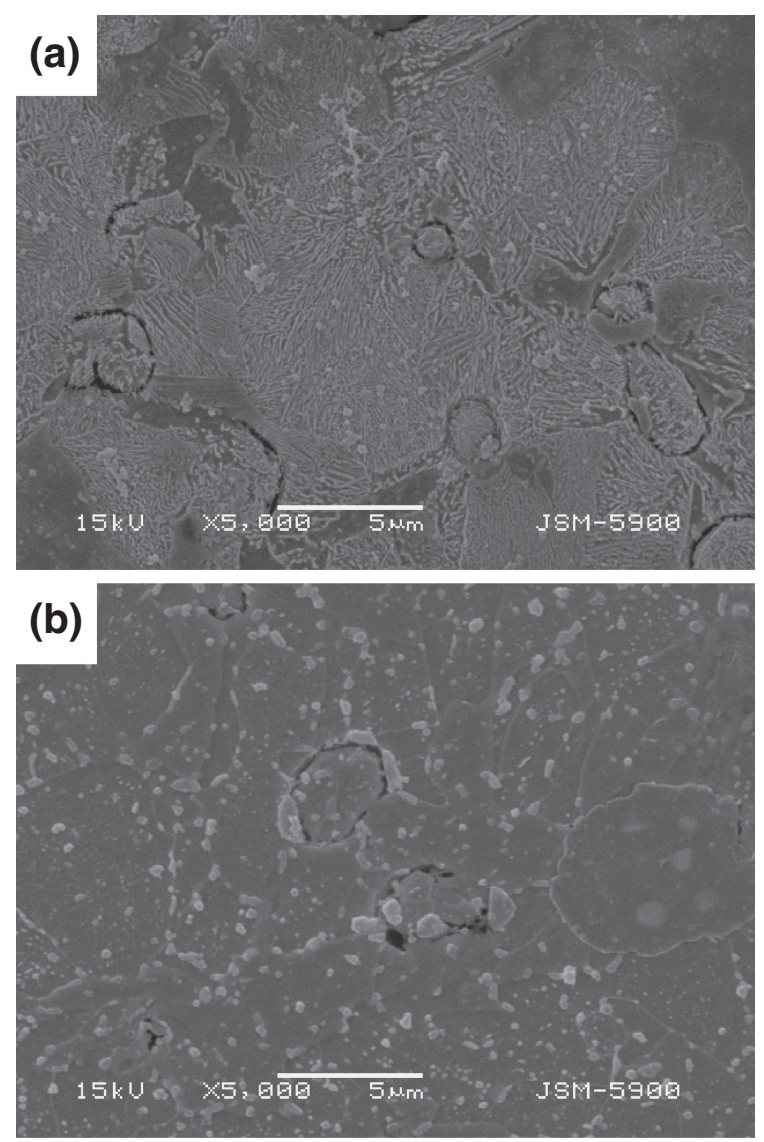

Fig. 1 SEM micrographs of (a) as-quenched and (b) tempered samples at $550^{\circ} \mathrm{C}$ for $600 \mathrm{~min}$.

$$
\begin{aligned}
\rho_{\alpha}= & (1.270 \mathrm{Fe}+1.380 \mathrm{C}+1.524 \mathrm{Mn}+2.381 \mathrm{Si} \\
& +1.370 \mathrm{Ni}+1.384 \mathrm{Cr}+1.076 \mathrm{Mo})^{-1} \times 10^{3}
\end{aligned}
$$

where the amount of each alloy is in mass $\%$. To obtain a fully martensitic microstructure, the specimens were annealed at $850^{\circ} \mathrm{C}$, where austenite is only stable for $30 \mathrm{~min}$, and immediately quenched in water. Subsequently, the specimens were tempered at 12 different conditions with a combination of temperatures $\left(250,350,450\right.$, and $\left.550^{\circ} \mathrm{C}\right)$ and times $(6,60$, and $600 \mathrm{~min})$. These tempering conditions were compared with experimental data taken from the literature. ${ }^{13)}$ After tempering, the samples were polished to a fine surface finish, etched using a $2 \%$ nital solution, and observed using a scanning electron microscope (SEM, JMS-5099). The average hardness value of the tempered martensite in each specimen was obtained from 10 Vickers hardness tests per specimen, excluding the minimum and maximum values.

\section{Results and Discussion}

Figure 1 shows the SEM micrographs of the as-quenched and tempered samples after the sintering process. The microstructure consists of martensite and pores can be seen in the as-quenched sample, as shown in Fig. 1(a). The cooling rate of water quenching, from a single austenite region of $850^{\circ} \mathrm{C}$, was fast enough to avoid other diffusional transformations until austenite decomposed to martensite. The small particles seem to be cementite carbides that were formed during the martensite transformation due to auto-
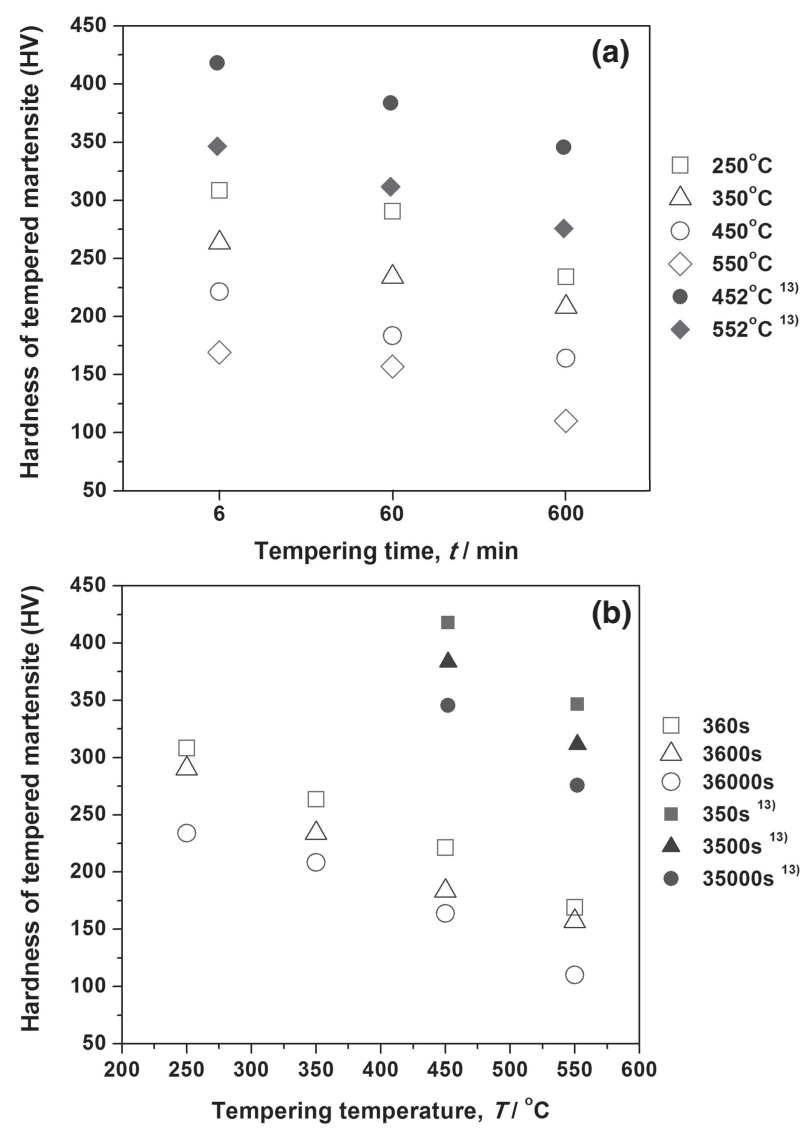

Fig. 2 Variation of hardness of tempered martensite according to (a) tempering time and (b) tempering temperature in a sintered $\mathrm{S} 55 \mathrm{C}$ steel compared with the hardness of tempered martensite in fully dense S55C. ${ }^{13)}$

tempering. A relatively high martensite start $\left(M_{\mathrm{S}}\right)$ temperature is required for the occurrence of auto-tempering; thus, the carbon content in the steel should be low (less than 0.2 mass \%). ${ }^{15)}$ Warke et al. reported that the $M_{\mathrm{s}}$ temperature increased as the relative density in the sintered PM steel decreased. ${ }^{16)}$ Thus, the occurrence of auto-tempering during quenching of the sintered S55C sample is possible although the carbon content in the S55C sample $(0.55$ mass $\%)$ is high enough to lower the $M_{\mathrm{s}}$ temperature and to suppress the occurrence of auto-tempering. Figure 1(b) shows the microstructure of tempered martensite with numerous carbide particles, which are uniformly dispersed in the matrix after tempering at $550^{\circ} \mathrm{C}$ for $600 \mathrm{~min}$. These carbide particles are cementite and have an average size smaller than $2 \sim 3 \mu \mathrm{m}$. This is the case because the sample contains no carbide precipitation alloy elements such as chromium or molybdenum.

Figures 2(a) and 2(b) show the variation of the tempered martensite hardness of S55C samples as a function of tempering condition and density. The closed symbols indicate the experimental data of fully dense S55C specimens taken from the literature, ${ }^{13}$ ) whereas the open symbols denote experimental values of sintered S55C specimens with $90 \%$ density. While the hardness values in both bulk and sintered specimens decrease with increasing tempering temperature and holding time, the sintered specimen values were $\sim 150$ to $200 \mathrm{HV}$ lower than the values of the bulk specimens. 
Typically, the mechanical properties, such as yield stress, tensile stress, hardness, elongation, and reduction of area, deteriorate rapidly as the relative density of the sintered metal alloys decrease. Wang reported that increasing the compacting pressure resulted in an increased hardness for sintered Fe-C-Cu-P alloys, caused by an increased relative density. ${ }^{17)}$ Also, Chawla and Deng found out that tensile strength, fatigue strength, elongation, and Young's modulus all increased as the porosity in sintered Fe-0.85Mo-Ni steels decreased. ${ }^{18)}$

Regardless of the degree of sintering, virgin martensite that is formed by quenching is softened during the tempering of steels. Thermally enriched carbon atoms in the octahedral sites of quenched martensite can diffuse and contribute to the formation of several carbides, depending on the tempering temperature. Thus, the tempering process is considered to be a diffusion-controlled phenomenon and is influenced by temperature and time. Hollomon and Jaffe proposed a simple tempering parameter to express the variation of tempered martensite hardness. ${ }^{1)}$ This parameter consists of the tempering temperature, isothermal tempering time, and activation energy for tempering. Waterschoot et al. showed that the activation energy of tempering is affected by the tempering condition and alloy composition due to carbide formation at different tempering stages. ${ }^{19)}$

Inoue used the $\lambda$-parameter, which is a different type of tempering parameter, to calculate the activation energy for tempering. ${ }^{20)}$ Tamaki and Suzuki also adopted this calculation method to compare the activation energies for several alloy steels during tempering. ${ }^{13)}$ The $\lambda$-parameter can be written as follows:

$$
\lambda=\log t-Q / 2.3 R(1 / T)+50
$$

where $t$ is the isothermal tempering time, $T$ is the tempering temperature in Kelvin, $R$ is the gas constant $(1.987 \mathrm{cal} / \mathrm{mol} /$ $\mathrm{K})$, and $Q$ is the activation energy for tempering. The previous researchers who used eq. (2) revealed that the hardness of tempered martensite $\left(H_{\mathrm{TM}}\right)$ was linearly proportional to the $\lambda$-parameter. The following relationship was proposed:

$$
H_{\mathrm{TM}}=m \lambda+n
$$

where $m$ and $n$ are constants. By combining eqs. (2) and (3), the experimental values of the tempered martensite hardness can be plotted versus the reciprocal of absolute temperature, and the activation energy of tempering is calculated from the slope of:

$$
H_{\mathrm{TM}}=-m Q / 2.3 R(1 / T)+(m \log t+50 m+n)
$$

The hardness values of tempered martensite in both bulk and sintered S55C samples are plotted against the $\lambda$-parameter in Fig. 3. Each set of hardness values for the different samples shows a linear relationship with the $\lambda$-parameter, as indicated in eq. (3). With the use of eq. (4), the effect of density on the activation energy for tempering is clearly confirmed: $58.83 \mathrm{kcal} / \mathrm{mol}$ for the bulk specimen $\left(100 \%\right.$ dense, $\left.Q_{100}\right)$ and $27.68 \mathrm{kcal} / \mathrm{mol}$ for the sintered specimen $(90 \%$ dense, $\left.Q_{90}\right)$.

Carbon atoms are supersaturated as a solid solution state in martensite, and the carbon atoms are rejected from the

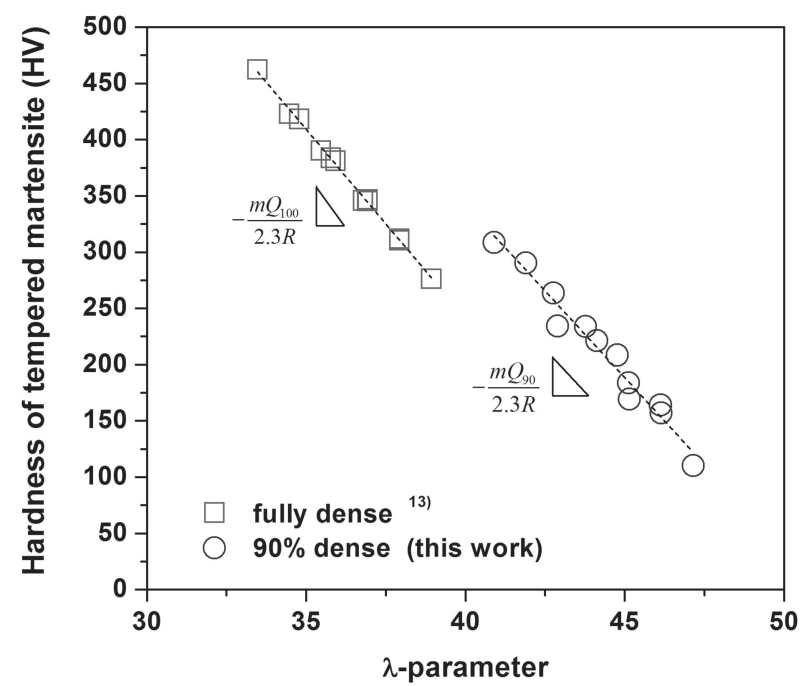

Fig. 3 Linear relationship between the hardness of tempered martensite and the $\lambda$-parameter of fully dense and sintered S55C specimens.

martensite by the formation of fine carbides during the tempering process. Therefore, the diffusion of carbon atoms in the martensite is a potent governing parameter of the tempering process. ${ }^{21)}$ Gallo et al. reported that the apparent diffusion coefficient of carbon atoms in sintered specimens is dependent on the density of the specimen as: ${ }^{22)}$

$$
D \cdot \rho^{x}=y
$$

where $D$ is the apparent diffusion coefficient of carbon in the sintered specimen, $\rho$ is the relative density of the sintered ferrous alloy, and $x$ and $y$ are constants depending on the processing conditions. ${ }^{23)}$ Khraisat and Nyborg also showed that $D$ decreases with increasing $\rho$ in sintered steel. ${ }^{24)}$ It could be inferred that the decrease in density of the sintered specimen in the present study may accelerate the diffusion of carbon atoms in the martensite during tempering, resulting in the acceleration of the tempering process. Namely, the reduced activation energy for the sintered specimen was derived from the lower density.

The tempering parameter $(T P)$, representing the extent of tempering proposed by Hollomon and Jaffe, ${ }^{1)}$ has been widely used as a critical parameter to predict the hardness of tempered martensite:

$$
T P=T(\log t+C)
$$

where $C$ is a material constant. A value of 20 is commonly adopted for $C$ (e.g., for dual-phase steels), ${ }^{7)}$ but the value is influenced by the composition of the alloy steels. Säglitz et al. used a $C$ value of 11.8 for low-carbon boron steel. $\left.{ }^{6}\right)$ Hollomon and Jaffe found that increasing the carbon content leads to a linear decrease in the $C$ value. ${ }^{1)}$ Also, Tamaki and Suzuki reported that the $C$ value was affected by both the carbon content and the amount of other alloying elements that are associated with the activation energy for tempering. ${ }^{13)}$ Recently, Kang and Lee proposed a compositiondependent tempering parameter. ${ }^{8)}$ By extensively analyzing experimental data in the literature, they concluded that the addition of alloying elements, such as $\mathrm{C}, \mathrm{Mn}, \mathrm{Si}, \mathrm{Ni}, \mathrm{Cr}$, and Mo, resulted in a decrease of the tempering parameter. 


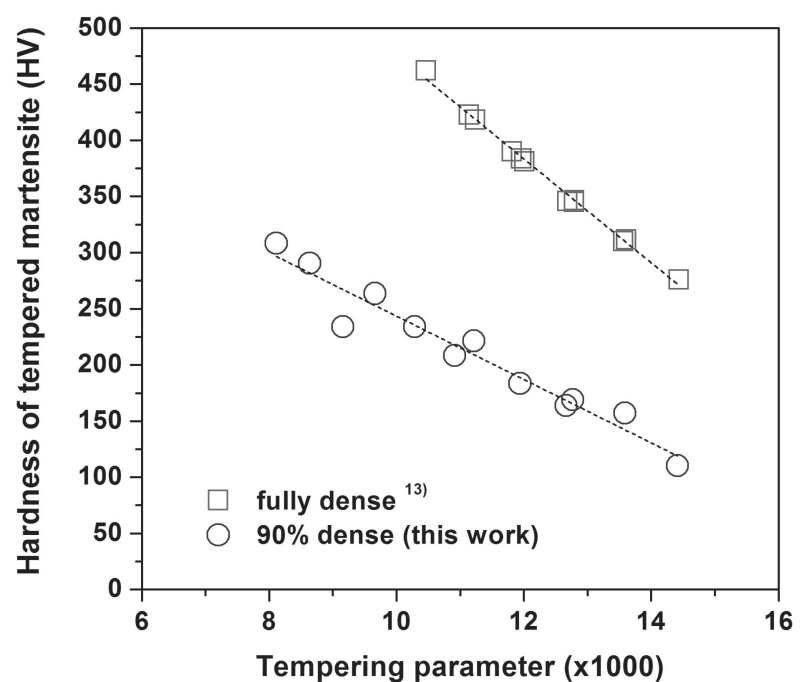

Fig. 4 Comparison of the tempered martensite hardness with the tempering parameter with a density-independent $C$ value (12.958).

However, few quantitative studies have been conducted on the density effect on the hardness of tempered martensite.

For a deeper understanding of the effect of density on the tempered martensite hardness, the hardness values are plotted against the $T P$, as shown in Fig. 4. Equation (6) was used to determine the $T P$ value. For this calculation, the $C$ value of 12.958 , obtained from the experimental data of the fully dense S55C sample, was used. A lower TP value represents a tempering condition with a relatively lower tempering temperature and a shorter holding time, which results in a higher hardness value. Because the experimental tempering condition in the present study was designed to be almost identical to that of Tamaki and Suzuki's previous work, ${ }^{13)}$ the effect of density on the hardness of tempered martensite was examined in the present study. The tempered martensite hardness of the sintered sample is obviously lower than that of the fully dense sample. Each range of TP values for the two samples are not overlapped because the tempering conditions are somewhat different. However, if the material density only contributes to the reduction of the hardness of tempered martensite, without influencing the $T P$, each slope of the data points for the two samples should be parallel.

The hardness of tempered martensite is gradually decreased as the tempering process is progressed. As plotting the hardness of tempered martensite varied with the TP the values are decreased linearly or parabolically depending on experimental results. Among the several possible types of empirical equations to express the relationship between the $T P$ and the hardness of tempered martensite, the following equation was selected because the hardness values of tempered martensite decreased linearly with increasing $T P$ values, as seen in Fig. 4:

$$
H_{\mathrm{TM}}=\alpha \times T P+\beta
$$

where $\alpha$ and $\beta$ are constants determined by fitting the experimental data. In the case of the fully dense S55C alloy, the optimized values of $\alpha$ and $\beta$ are -0.0458 and +932.452 , respectively. Figure 5 compares the predicted hardness values of tempered martensite, using the optimal $\alpha$ and $\beta$

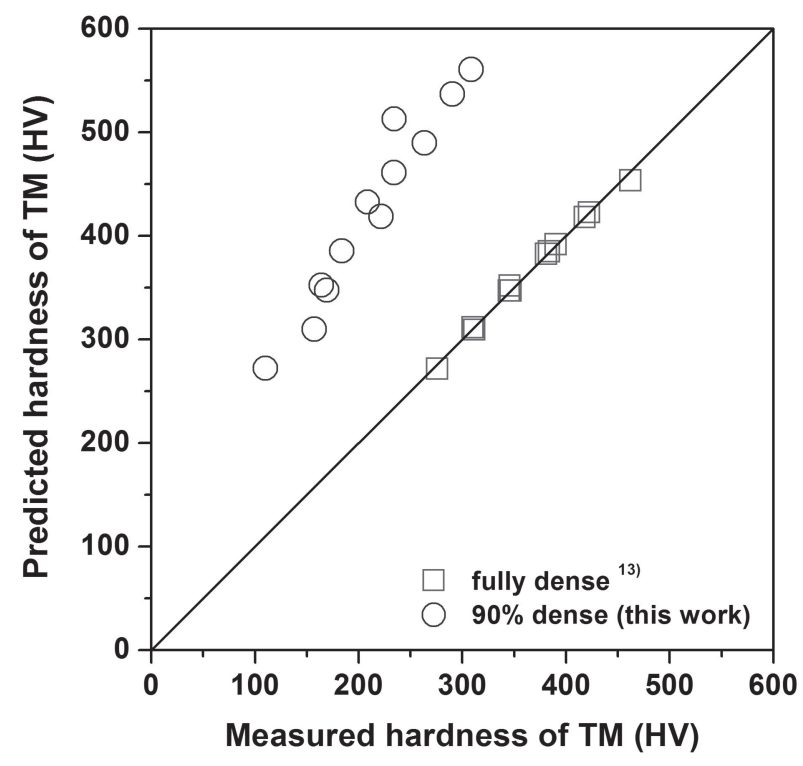

Fig. 5 Predicted hardness of tempered martensite (TM) using eq. (7) with the density-independent $C$ value (12.958) in the tempering parameter compared with experimental data. ${ }^{13)}$

values for the fully dense S55C alloy and the $C$ value of 12.958 , with the measured data. While the predicted hardness values of the bulk sample matched well with measured values, those of the sintered sample are higher than the experimental data. This overestimation is explained as an extension of the relation between the $T P$ and the existence of porosity, as discussed in Fig. 4.

Since the activation energy for tempering appears to decrease as the density of the sintered specimen decreases, via the accelerated diffusion of carbon atoms in the martensite during tempering, the existence of porosity influences the $C$ value in the $T P$. The optimized $C$ value obtained from the sintered S55C sample was 6.098, whereas the $C$ value for the fully dense S55C sample was 12.958 . This implies that the density affects the activation energy for tempering and causes a drastic reduction of the $T P$ values. Thus, the hardness values are plotted against the $T P$ with the distinguished $C$ values (which rely on the density effect seen in Fig. 6). When compared with the data distribution in Fig. 4, the $T P$ values for the sintered sample become much smaller and the range of the $T P$ values is reduced, resulting in parallel slopes of the data points for the two samples.

Based on the analysis of the experimental results, we propose the empirical equation for predicting the hardness of tempered martensite, using the tempering parameter which accounts for the density-dependent $C$ function, to be:

$$
\begin{aligned}
H_{\mathrm{TM}}= & -0.0458 \times[T(\log t+(12.272+0.686 d))] \\
& +(890.161+42.291 d)
\end{aligned}
$$

where $H_{\mathrm{TM}}$ is the tempered martensite hardness in Vickers, $T$ is the tempering temperature in Kelvin, $t$ is the isothermal tempering time in seconds, and $d$ is the degree of density (\%). The suggested equation includes two important effects of density: the $T P$ shifting effect via reduction of the $C$ value associated with the activation energy for tempering and the effect of decreasing hardness due to the existence of porosity. As shown in Fig. 7, the predicted tempered martensite 


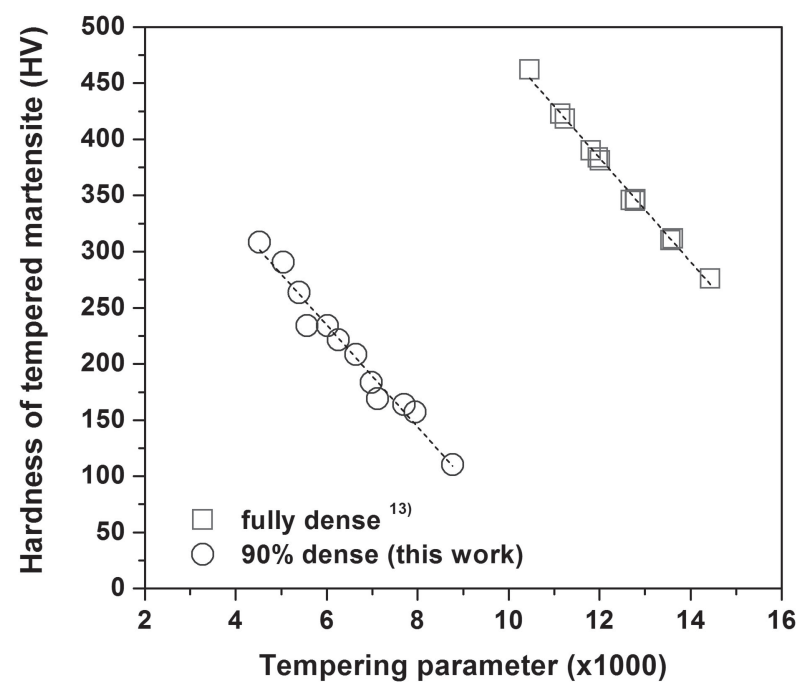

Fig. 6 Comparison of the tempered martensite hardness with the tempering parameter with a density-dependent $C$ value.

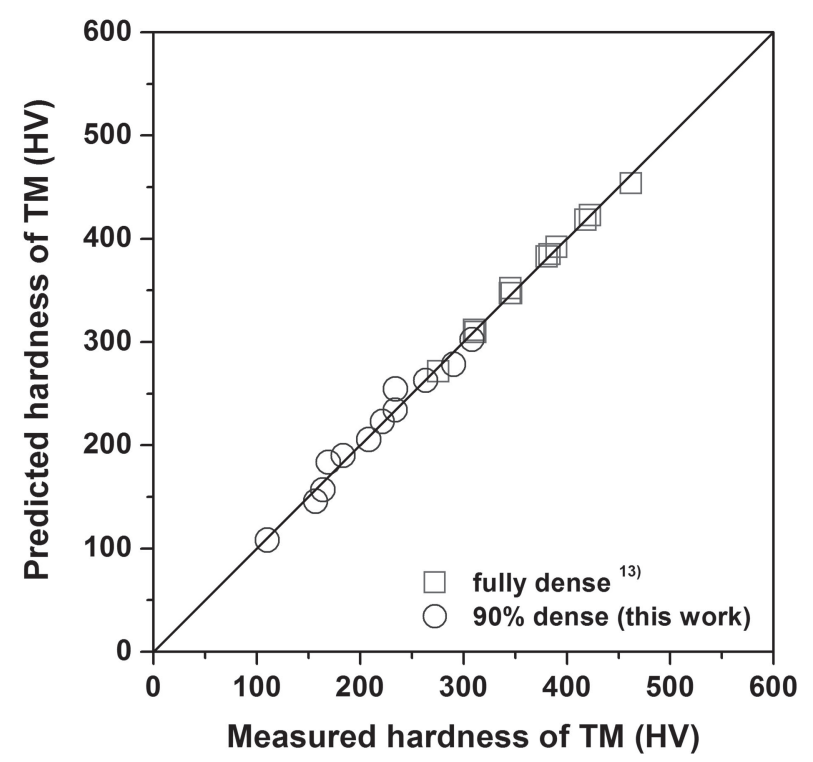

Fig. 7 Predicted hardness of tempered martensite (TM) using eq. (8) with the density-dependent $C$ value in the tempering parameter compared with experimental data. ${ }^{13)}$

hardness, which uses the proposed equation, shows good agreement with the experimental data that includes the density effect. It is anticipated that the proposed equation considering the density effect will be applied to other sintered alloy steels.

\section{Conclusions}

An empirical equation for calculating the hardness of tempered martensite that takes into account the density effect was proposed by comparing fully dense S55C data obtained from the literature with sintered $\mathrm{S} 55 \mathrm{C}$ data obtained experimentally. The calculated activation energy for tempering was decreased as the relative density decreased due to the fact that the decrease of density accelerated the diffusion of carbon atoms in the tempered martensite of the sintered carbon steel. This led to an accelerated tempering process that appears to have reduced the activation energy required for tempering. The existence of porosity not only decreased the hardness but also shifted the TP; the influence of these effects is adopted in the proposed equation. By using the densitydependent $C$ value in the $T P$, we achieved good agreement with the experimental data obtained under various tempering conditions and relative densities. We anticipate that our newly proposed equation can be used to determine the tempering conditions required to achieve a target hardness value of tempered martensite in sintered steels.

\section{REFERENCES}

1) J. H. Hollomon and L. D. Jaffe: Trans. AIME 162 (1945) 223-249.

2) J. P. Materkowski and G. Krauss: Metall. Mater. Trans. A 10 (1979) 1643-1651.

3) K. Suzuki, I. Kurihara, T. Sasaki, Y. Koyoma and Y. Tanaka: Nuc. Eng. Des. 206 (2001) 261-277.

4) H. K. D. H. Bhadeshia and R. W. K. Honeycombe: Steels. Microstructure and Properties, third ed., (Elsevier Ltd., Oxford; 2006) pp. 183-208.

5) E. Virtanen, C. J. Van Tyne, B. S. Levy and G. Brada: J. Mater. Proc. Technol. 213 (2013) 1364-1369.

6) M. Säglitz, D. K. Matlock and G. Krauss: Proc. of Int. Conf. on New Developments in Advanced High Strength Steels, (AIST, Warrendale, PA, 2008) pp. 147-154.

7) A. Kamp, S. Celotto and D. N. Hanlon: Mater. Sci. Eng. A 538 (2012) 35-41.

8) S. Kang and S. J. Lee: Mater. Trans. 55 (2014) 1069-1072.

9) K. S. Narasimhan: Adv. Perform. Mater. 3 (1996) 7-27.

10) R. Chandramouli, T. K. Kandavel, D. Shanmugasundaram and T. Ashok Kumar: Mater. Des. 28 (2007) 2260-2264.

11) M. Behnam, A. S. Golezani and M. M. Lima: Powder Technol. 235 (2013) 1025-1029

12) S. Narayan and A. Rajeshkannan: Mater. Res. 15 (2012) 291-299.

13) K. Tamaki and J. Suzuki: Res. Rep. Fac. Eng. Mie Univ. 15 (1990) 922.

14) U. Bohnenkamp and R. Sandström: Steel Res. 71 (2000) 88-93.

15) Q. Han, Y. Kang, X. Zhao, N. Stanford and M. Cai: Mater. Des. 51 (2013) 409-414.

16) V. S. Warke, R. D. Sisson, Jr. and M. M. Makhlouf: Metall. Mater. Trans. A 40 (2009) 569-572.

17) W. F. Wang: Mater. Sci. Eng. A 402 (2005) 92-97.

18) N. Chawla and X. Deng: Mater. Sci. Eng. A 390 (2005) 98-112.

19) T. Waterschoot, K. Verbeken and B. C. De Cooman: ISIJ Int. 46 (2006) $138-146$.

20) T. Inoue: Tetsu-to-Hagane 66 (1980) 1532-1541.

21) M. Perez, C. Sidoroff, A. Vincent and C. Esnouf: Acta Mater. 57 (2009) 3170-3181.

22) A. Gallo, A. Elia and S. Ciambellini: Powder Metall. Int. 16 (1984) 177-179.

23) G. F. Bocchini: Powder Metall. Prog. 4 (2004) 1-34.

24) W. Khraisat and L. Nyborg: Mater. Sci. Technol. 20 (2004) 705-710. 